\title{
Impact of gauge fixing on angular momentum operators of the covariantly quantized electromagnetic field
}

\author{
Bogdan Damski \\ Jagiellonian University, Institute of Theoretical Physics, Łojasiewicza 11, 30-348 Kraków, Poland
}

(Received 13 May 2021; accepted 29 July 2021; published 1 October 2021)

\begin{abstract}
Covariant quantization of the electromagnetic field imposes the so-called gauge-fixing modification on the Lagrangian density. As a result of that, the total angular momentum operator receives at least one gaugefixing-originated contribution, whose presence causes some confusion in the literature. The goal of this work is to discuss in detail why such a contribution, having no classical interpretation, is actually indispensable. For this purpose, we divide canonical and Belinfante-Rosenfeld total angular momentum operators into different components and study their commutation relations, their role in the generation of rotations of quantum fields, and their action on states from the physical sector of the theory. Then, we examine physical matrix elements of operators having gauge-fixing-related contributions, illustrating problems that one may encounter due to careless employment of the resolution of identity during their evaluation. The resolution of identity, in the indefinite-metric space of the covariantly quantized electromagnetic field, is extensively discussed because it takes a not-so-intuitive form if one insists on explicit projection onto states from the physical sector of the theory. Our studies are carried out in the framework of the Gupta-Bleuler theory of the free electromagnetic field. Relevant remarks about interacting systems, described by covariantly quantized electrodynamics, are given.
\end{abstract}

DOI: 10.1103/PhysRevD.104.085003

\section{INTRODUCTION}

We all know that covariantly quantized electrodynamics has some appealing features. One is that its free-field photon propagator has the simplest possible form, which facilitates perturbative calculations. The other is that its electromagnetic vector potential $A^{\mu}$ is manifestly Lorentz covariant, which comes in handy when relativistic transformation properties of operators and matrix elements involving $A^{\mu}$ are discussed. In addition to that, such $A^{\mu}$ is local, which is important in certain rigorous studies of quantum field theory (see e.g. Sec. 1.1 of [1] for relevant references). These and related convenient features of the covariant quantization framework do not come for free. The price one has to pay for them can be seen in different ways.

To begin, one has to accept the fact that unphysical degrees of freedom are being introduced by the quantization procedure, scalar and longitudinal photons, critically commented upon by Schwinger [2], Strocchi [3], and others. To make matters worse, the abnormal commutation relation of creation and annihilation operators of scalar photons leads to the conclusion that $A^{\mu}$ is defined on the

Published by the American Physical Society under the terms of the Creative Commons Attribution 4.0 International license. Further distribution of this work must maintain attribution to the author(s) and the published article's title, journal citation, and DOI. Funded by SCOAP . indefinite-metric space [4,5], where vectors can have positive, negative, or zero norm [6].

This result asks for the definition of the physically relevant sector of such a space, and for this purpose one typically assumes that matrix elements of $\partial \cdot A$ vanish in states from such a subspace. The very existence of such a condition strikingly illustrates problems with enforcement of the Lorenz gauge on the operator level, which can be seen as one more complication. These rather unwelcome features were technically addressed in early papers of Gupta [7] and Bleuler [8], quantizing the electromagnetic field in the Feynman gauge (see [9] for the comprehensive summary of these efforts). Those studies were subsequently generalized to account for other covariant gaugesespecially the Landau gauge-by Lautrup [10] and Nakanishi [5] (see Sec. 18 of [5] for comments about [10], Sec. 7 of [11] for textbook discussion of [10], and [12] for a recent overview).

As far as this work is concerned, the main technical complication of the covariant quantization approach is that it modifies the Lagrangian density by adding the socalled gauge-fixing term to it. The most elementary reason for doing so is that it solves the problem of vanishing momentum canonically conjugate to $A^{0}$. This allows for quantization of all components of the electromagnetic vector potential, which is of key importance in the covariant quantization framework. The gauge-fixing modification of the Lagrangian density can be also seen as a Lagrange 
multiplier, imposing the Landau gauge in the appropriate limit (see e.g. Sec. 15.5 of [13]).

Such a procedure leads to field equations that do not correspond to Maxwell (Maxwell-Dirac) equations in free (interacting) systems. Interestingly, this complication can be, in the free theory of the electromagnetic field, rigorously linked to locality and Lorentz covariance of $A^{\mu}$ (these properties are necessarily lost when Maxwell's equations are kept as operator identities $[3,14])$.

The change of the Lagrangian density automatically affects various observables derived from it. This remark brings our attention to conserved quantities in general and angular momentum, being of special interest here, in particular. Namely, the total angular momentum operator receives at least one gauge-fixing correction, which causes some confusion.

The confusion arises when one (i) starts from the classical theory, which neither needs nor has any gaugefixing modifications, (ii) derives the classical expression for total angular momentum, (iii) replaces the classical electromagnetic vector potential in it by the covariantly quantized one, and (iv) assumes that the resulting expression represents the total angular momentum operator.

In general, such a procedure leads to the expression violating some of the most basic properties expected of the total angular momentum operator. Its failure can be traced back to the (iii) step, which is done under the tacit assumption that the covariant quantization can be carried out without the gauge-fixing modification of the Lagrangian density. This is not the case, which we have already mentioned. To fix the above procedure, it is sufficient to alter the (i) step by working from the very beginning with the modified Lagrangian density, inevitably introducing gaugefixing angular momentum to the theory. The purpose of this work is to discuss in detail why such an awkward kind of angular momentum, completely redundant in the classical theory, is actually indispensable on the quantum level.

This can be done in the most transparent manner in the free theory of the electromagnetic field, which we will be exploring in the following sections (relevant remarks about the interacting fields will be also given). Our discussion will be focused on the two most popular ways in which the (ii) step is performed: the canonical and BelinfanteRosenfeld decompositions.

These and other decompositions, frequently commented upon in literature $[15,16]$, aim at division of the total angular momentum operator into physically meaningful contributions. The following question then arises. What is the role of the gauge-fixing angular momentum operator in such considerations?

Given substantial interest in angular momentum-related physics, it is a bit surprising that this issue is poorly explored in the literature. For example, nothing can be found about the gauge-fixing angular momentum operator in popular review [16] and numerous other works tacitly assuming that the gauge-fixing procedure is absent, while at the same time intensely discussing the division of the total angular momentum operator into gauge-invariant components [17]. These considerations do not apply to covariantly quantized theories, such as the Feynman gauge one that we study, because they are carried out irrespective of the quantization procedure necessarily bringing the gauge-fixing angular momentum operator to the table.

The inescapable fact of its presence, in studies assuming that the operator $A^{\mu}$ transforms as a genuine 4-vector, was stated in [18]. In particular, one can find in this article interesting critical assessment of papers ignoring gauge-fixing-originated contributions to total linear and angular momentum operators. In this context, our work comprehensively illustrates the importance of the latter contributions, approaching the subject from a different perspective than [18].

If one acknowledges that there are gauge-fixingoriginated contributions to various observables in a covariantly quantized theory, then it is of interest to know whether they contribute to physical matrix elements (i.e. the ones computed in states from the physically relevant sector of the theory). This issue was discussed in [18] for the density of the energy-momentum tensor. It was argued there that its gauge-fixing-related part has vanishing physical matrix elements, and then this statement was reiterated in a popular review [15]. However, the argument leading to such a conclusion relies on insertion of a complete set of physical states between field operators in the considered matrix elements [18]. We question validity of such an argument, supporting our view by a detailed discussion of the subtle issues associated with the resolution of identity in the indefinite-metric space.

Before presenting the outline of this work, we mention that the gauge-fixing modification of the Lagrangian density can be avoided when one works in the noncovariant gauge that is effected on the operator level. For example, this is the case in the Coulomb gauge studies, if one parametrizes the spatial part of the electromagnetic vector potential operator so as to satisfy the $\operatorname{div} \boldsymbol{A}=0$ constraint [11]. Then clearly some aspects of angular momentum-related studies are simpler. However, the pleasant features of the covariant quantization framework, including the ones mentioned at the beginning of this section, are lost. This is why we believe that it is worth to face subtleties of the covariant approach head on. Moreover, we mention that it is our opinion that covariant gauge studies of angular momentum physics are underrepresented in the literature, which additionally motivates us for pursuing them. Finally, it should be said that at least some noncovariant gauges can be also implemented by the gauge-fixing modification of the Lagrangian density (see e.g. [19] for the Coulomb gauge example). Its influence on the total angular momentum operator could be presumably analyzed akin to what we do in this work. 
The outline of this paper is the following. Section II briefly presents classical results on angular momentum of the electromagnetic field from the perspective relevant to our studies. Section III is devoted to quantum investigations of the canonical decomposition of the total angular momentum operator. It starts, however, with the introduction to the Gupta-Bleuler quantization scheme, before focusing on three components of such an operator. Their commutation relations, their role in the generation of rotations of quantum fields, and their action on states from the physical sector of the theory are extensively discussed in Secs. III A, III B, and III C, respectively. Analogical analysis is then presented in Sec. IV for the BelinfanteRosenfeld decomposition. Next, physical matrix elements of gauge-fixing-related operators are discussed in Sec. V. Particular stress is placed there upon presentation of the correct form of the resolution of identity in the indefinitemetric space because it takes a not-so-intuitive form if one insists on explicit projection onto states from the physical sector of the theory. This discussion is then continued in Appendix. The summary of our work is given in Sec. VI.

\section{CLASSICAL CONSIDERATIONS}

The classical Lagrangian density for the free electromagnetic field is given by

$$
\mathcal{L}^{\mathrm{cl}}=-\frac{1}{4} F_{\mu \nu}^{\mathrm{cl}} F_{\mathrm{cl}}^{\mu \nu},
$$

where $F_{\mu \nu}^{\mathrm{cl}}=\partial_{\mu} A_{\nu}^{\mathrm{cl}}-\partial_{\nu} A_{\mu}^{\mathrm{cl}}$ and the symbol cl is used to distinguish classical expressions from their quantum counterparts studied in the following sections. To specify the remaining conventions, we mention that we adopt the Heaviside-Lorentz system of units and set $\hbar=c=1$. Moreover, we use the metric tensor $\eta=\operatorname{diag}(+---)$ and assume that Greek and Latin indices of tensors take values $0,1,2,3$ and 1,2,3, respectively. The Einstein summation convention is frequently applied to them. 3 -vectors are written in bold, e.g. $x=\left(x^{\mu}\right)=\left(x^{0}, \boldsymbol{x}\right)$. The Levi-Civita symbol is written as $\varepsilon^{i j k}$, where $\varepsilon^{123}=+1$.

To obtain the canonical expression for angular momentum, one evaluates [11]

$$
\begin{aligned}
J_{\mathrm{cl}}^{i} & =\frac{1}{2} \varepsilon^{i m n} \int d^{3} z\left(\vartheta_{\mathrm{cl}}^{0 n} z^{m}-\vartheta_{\mathrm{cl}}^{0 m} z^{n}+\delta M_{\mathrm{cl}}^{0 m n}\right), \\
\vartheta_{\mathrm{cl}}^{\mu \nu} & =\frac{\partial \mathcal{L}^{\mathrm{cl}}}{\partial\left(\partial_{\mu} A_{\mathrm{cl}}^{\sigma}\right)} \partial^{\nu} A_{\mathrm{cl}}^{\sigma}-\eta^{\mu \nu} \mathcal{L}^{\mathrm{cl}}, \\
\delta M_{\mathrm{cl}}^{\mu \nu \lambda} & =\frac{\partial \mathcal{L}^{\mathrm{cl}}}{\partial\left(\partial_{\mu} A_{\mathrm{cl}}^{\sigma}\right)}\left(I^{\nu \lambda}\right)^{\sigma \rho} A_{\rho}^{\mathrm{cl}} \\
\left(I^{\alpha \beta}\right)^{\gamma \delta} & =\eta^{\alpha \gamma} \eta^{\beta \delta}-\eta^{\alpha \delta} \eta^{\beta \gamma}
\end{aligned}
$$

where $\vartheta_{\mathrm{cl}}^{\mu \nu}$ stands for the canonical energy-momentum tensor density while $\delta M_{\mathrm{cl}}^{\mu \nu \lambda}$ is the so-called spin contribution. In the end, one finds that (2) has the following spin and orbital components:

$$
\begin{aligned}
\boldsymbol{J}^{\mathrm{cl}} & =\boldsymbol{J}_{\mathrm{spin}}^{\mathrm{cl}}+\boldsymbol{J}_{\mathrm{orb}}^{\mathrm{cl}}, \\
\boldsymbol{J}_{\text {spin }}^{\mathrm{cl}} & =\int d^{3} z \boldsymbol{E}^{\mathrm{cl}} \times \boldsymbol{A}^{\mathrm{cl}}, \\
\boldsymbol{J}_{\mathrm{orb}}^{\mathrm{cl}} & =\int d^{3} z E_{\mathrm{cl}}^{j}(z \times \nabla) A_{\mathrm{cl}}^{j},
\end{aligned}
$$

where neither Euler-Lagrange equations nor changes of the integrands through addition or subtraction of 3-divergence terms have been employed during derivation of (3).

A different procedure for defining angular momentum appears when one works with the $\mu \leftrightarrow \nu$ symmetric energymomentum tensor density [11]

$\tilde{\vartheta}_{\mathrm{cl}}^{\mu \nu}=\vartheta_{\mathrm{cl}}^{\mu \nu}+\partial_{\sigma} \chi_{\mathrm{cl}}^{\sigma \mu \nu}$,

$$
\begin{aligned}
\chi_{\mathrm{cl}}^{\sigma \mu \nu}= & \frac{A_{\lambda}^{\mathrm{cl}}}{2}\left[\frac{\partial \mathcal{L}^{\mathrm{cl}}}{\partial\left(\partial_{\sigma} A_{\mathrm{cl}}^{\rho}\right)}\left(I^{\mu \nu}\right)^{\rho \lambda}+\frac{\partial \mathcal{L}^{\mathrm{cl}}}{\partial\left(\partial_{\nu} A_{\mathrm{cl}}^{\rho}\right)}\left(I^{\mu \sigma}\right)^{\rho \lambda}\right. \\
& \left.-\frac{\partial \mathcal{L}^{\mathrm{cl}}}{\partial\left(\partial_{\mu} A_{\mathrm{cl}}^{\rho}\right)}\left(I^{\sigma \nu}\right)^{\rho \lambda}\right]
\end{aligned}
$$

which is obtained from (4) after employment of EulerLagrange equations. It is used for computation of angular momentum via

$$
\tilde{J}_{\mathrm{cl}}^{i}=\frac{1}{2} \varepsilon^{i m n} \int d^{3} z\left(\tilde{\vartheta}_{\mathrm{cl}}^{0 n} z^{m}-\tilde{\vartheta}_{\mathrm{cl}}^{0 m} z^{n}\right) .
$$

In the end, one arrives at the Belinfante-Rosenfeld expression [20-22]

$$
\tilde{\boldsymbol{J}}^{\mathrm{cl}}=\int d^{3} z \boldsymbol{z} \times\left(\boldsymbol{E}^{\mathrm{cl}} \times \boldsymbol{B}^{\mathrm{cl}}\right) .
$$

Thorough discussion of quantum equivalents of (3) and (6) will be presented in Secs. III and IV, respectively.

\section{QUANTUM CONSIDERATIONS: CANONICAL EXPRESSIONS}

Upon transition to the quantum theory, the classical vector potential $A_{\mathrm{cl}}^{\mu}$ gets replaced by the operator $A^{\mu}$, which may suggest that the total canonical angular momentum operator will be given by (3) stripped off the symbol cl. It is actually not what is happening, which we have already commented upon in Sec. I.

It has been also mentioned there that the theory based solely on Lagrangian density (1) cannot be covariantly 
quantized. This issue is resolved by adding the gaugefixing term to (1)

$$
\mathcal{L}^{\mathrm{cl}} \rightarrow \mathcal{L}=-\frac{1}{4} F_{\mu \nu} F^{\mu \nu}-\frac{1}{2}(\partial \cdot A)^{2},
$$

which leads to the following canonical commutation relations [11]:

$$
\begin{aligned}
{\left[A^{\mu}(x), A^{\nu}(y)\right] } & =0, \\
{\left[\partial_{0} A^{\mu}(x), A^{\nu}(y)\right] } & =\mathrm{i} \eta^{\mu \nu} \delta(\boldsymbol{x}-\boldsymbol{y}), \\
{\left[\partial_{0} A^{\mu}(x), \partial_{0} A^{\nu}(y)\right] } & =0,
\end{aligned}
$$

where equal times, $x^{0}=y^{0}$, are assumed [23].

The vector potential operator then reads

$$
\begin{aligned}
A^{\mu}(z) & =\int \frac{d^{3} k}{(2 \pi)^{3 / 2}} \sum_{\sigma=0}^{3} \frac{\epsilon^{\mu}(\boldsymbol{k}, \sigma) c_{\boldsymbol{k} \sigma}}{\sqrt{2 \omega_{\boldsymbol{k}}}} e^{-\mathrm{i} k \cdot z}+\text { H.c., } \\
{\left[c_{\boldsymbol{k} \sigma}, c_{\boldsymbol{k}^{\prime} \sigma^{\prime}}^{\dagger}\right] } & =-\eta_{\sigma \sigma^{\prime}} \delta\left(\boldsymbol{k}-\boldsymbol{k}^{\prime}\right), \\
{\left[c_{\boldsymbol{k} \sigma}, c_{\boldsymbol{k}^{\prime} \sigma^{\prime}}\right] } & =0
\end{aligned}
$$

where $\left(k^{\mu}\right)=\left(\omega_{k}, \boldsymbol{k}\right), \omega_{\boldsymbol{k}}=|\boldsymbol{k}|$, and H.c. stands for the Hermitian conjugation [24]. Moreover, the polarization 4-vectors satisfy completeness and orthonormality relations

$$
\begin{gathered}
\sum_{\sigma=0}^{3} \eta_{\sigma \sigma} \epsilon^{\mu}(\boldsymbol{k}, \sigma) \epsilon^{\nu}(\boldsymbol{k}, \sigma)=\eta^{\mu \nu}, \\
\epsilon(\boldsymbol{k}, \sigma) \cdot \epsilon\left(\boldsymbol{k}, \sigma^{\prime}\right)=\eta_{\sigma \sigma^{\prime}} .
\end{gathered}
$$

They are chosen such that for transverse polarizations $\left(\sigma, \sigma^{\prime}=1,2\right)$

$$
\begin{gathered}
\epsilon(\boldsymbol{k}, \sigma)=(0, \boldsymbol{\epsilon}(\boldsymbol{k}, \sigma)), \\
\boldsymbol{\epsilon}(\boldsymbol{k}, \sigma) \cdot \boldsymbol{\epsilon}\left(\boldsymbol{k}, \sigma^{\prime}\right)=\delta_{\sigma \sigma^{\prime}}, \\
\boldsymbol{\epsilon}(\boldsymbol{k}, \sigma) \cdot \boldsymbol{k}=0,
\end{gathered}
$$

while for scalar $(\sigma=0)$ and longitudinal $(\sigma=3)$ ones

$$
\begin{gathered}
\epsilon(\boldsymbol{k}, 0)=(1, \mathbf{0}), \\
\epsilon(\boldsymbol{k}, 3)=\left(0, \boldsymbol{k} / \omega_{\boldsymbol{k}}\right) .
\end{gathered}
$$

We also mention that Fock states, obtained via the repeated action of creation operators $c_{k \sigma}^{\dagger}$ on the vacuum state $|0\rangle$, belong to the indefinite-metric space, say $\mathcal{H}$ from now on. It is so because the prefactor on the right-hand side of (9b) is negative for $\sigma=\sigma^{\prime}=0$.
The Lorenz gauge constraint, amounting classically to $\partial \cdot A_{\mathrm{cl}}=0$, now takes the form

$$
\left\langle\psi_{G B}|\partial \cdot A| \psi_{G B}^{\prime}\right\rangle=0 .
$$

This holds when

$$
L_{k}\left|\psi_{G B}\right\rangle=L_{k}\left|\psi_{G B}^{\prime}\right\rangle=0, \quad L_{k}=c_{k 3}-c_{k 0},
$$

which is supposed to happen for all 3-momenta $\boldsymbol{k}$ [25]. Such a condition follows from

$$
\partial \cdot A(z)=\mathrm{i} \int \frac{d^{3} k}{(2 \pi)^{3 / 2}} \sqrt{\frac{\omega_{k}}{2}} L_{k} e^{-\mathrm{i} k \cdot z}+\text { H.c. }
$$

States that are annihilated by operators $L_{\mathbf{k}}$ form the space $\mathcal{H}_{G B}$, where the subscript $G B$ refers to Gupta and Bleuler. The elements of $\mathcal{H}_{G B}$ can be written as linear combinations of the states

$$
\begin{aligned}
\left|\psi_{G B}\right\rangle= & \left|\psi_{T}\right\rangle+\int d^{3} k f(\boldsymbol{k}) L_{\boldsymbol{k}}^{\dagger}\left|\psi_{T}\right\rangle \\
& +\int d^{3} k d^{3} k^{\prime} f\left(\boldsymbol{k}, \boldsymbol{k}^{\prime}\right) L_{\boldsymbol{k}}^{\dagger} L_{\boldsymbol{k}^{\prime}}^{\dagger}\left|\psi_{T}\right\rangle+\cdots,
\end{aligned}
$$

where $f(\boldsymbol{k}), f\left(\boldsymbol{k}, \boldsymbol{k}^{\prime}\right)$, etc. are some functions and $\left|\psi_{T}\right\rangle$ is either the vacuum state or the state containing physical (transverse) photons only (Sec. 7.4 of [11]). The second, third, and the following terms on the righthand side of (20) have zero norm, which can be seen by combining (18) with

$$
\left[L_{k}, L_{k^{\prime}}^{\dagger}\right]=0 .
$$

Those states involve unphysical (scalar and longitudinal) photons. Their collection spans the space $\mathcal{H}_{0}$.

The physical states of the theory can be defined by elements of either $\mathcal{H}_{G B}$ or $\mathcal{H}_{G B} / \mathcal{H}_{0}$. The former definition, e.g., can be found in Sec. 18 of [5] and Sec. I.5.3 of [26]. The latter is employed in [27], discussing the concept of equivalence between those vectors from $\mathcal{H}_{G B}$, which differ by a zero-norm vector $\left[\left|\psi_{G B}\right\rangle\right.$ and $\left|\psi_{T}\right\rangle$ from (20) belong to the same equivalence class for any choice of functions $f$ ]. We adopt the former definition, calling all states from $\mathcal{H}_{G B}$ physical. We will see implementation of this terminology right below, where one more definition will be introduced.

The Hermitian operator $O$ will be labeled as physical when $O\left|\psi_{G B}\right\rangle \in \mathcal{H}_{G B}$ for all $\left|\psi_{G B}\right\rangle \in \mathcal{H}_{G B}$ [27]. This requirement amounts to saying that

$$
\left[L_{k}, O\right]\left|\psi_{G B}\right\rangle=0 .
$$

Having completed discussion of the covariant quantization of the electromagnetic field, we are ready for writing down its total canonical angular momentum operator 


$$
\begin{aligned}
\boldsymbol{J} & =\boldsymbol{J}_{\text {spin }}+\boldsymbol{J}_{\text {orb }}+\boldsymbol{J}_{\xi}, \\
J_{\text {spin }}^{i} & =\int d^{3} z \varepsilon^{i m n} F_{m 0} A_{n}, \\
J_{\text {orb }}^{i} & =\int d^{3} z \varepsilon^{i m n} z^{m} F_{j 0} \partial_{n} A_{j}, \\
J_{\xi}^{i} & =\int d^{3} z \varepsilon^{i m n} z^{m} \partial \cdot A \partial_{n} A_{0},
\end{aligned}
$$

which can be obtained by combining (2) with (7). The following comments are pertinent to these expressions.

First, the time argument of $\boldsymbol{J}_{\text {spin }}, \boldsymbol{J}_{\text {orb }}$, and $\boldsymbol{J}_{\boldsymbol{\xi}}$ is suppressed, which should not lead to any confusion as long as one remembers about remark [23]. We will proceed similarly with angular momentum operators discussed in Sec. IV.

Second, the order of operators on the right-hand sides of (23b)-(23d) does not matter, which can be shown with (9).

Third, we call $\boldsymbol{J}_{\xi}$ the gauge-fixing angular momentum operator. The subscript $\xi$ is used as it is customarily associated with covariant gauges. Note that such a type of angular momentum is absent in classical studies.

Fourth, we will refer to $\boldsymbol{J}_{\text {spin }}, \boldsymbol{J}_{\text {orb }}$, and $\boldsymbol{J}_{\boldsymbol{\xi}}$ as angular momentum operators (the same nomenclature will be applied to operators analyzed in Sec. IV). This is a slight abuse of terminology as none of them possesses all properties conventionally expected of a genuine angular momentum operator (e.g. none of them generates rotations of quantum fields). This will be discussed in detail in the following three subsections, where we will study various properties of these operators, paying special attention to the role of $\boldsymbol{J}_{\boldsymbol{\xi}}$ in making $\boldsymbol{J}$ a genuine angular momentum operator.

\section{A. Commutation relations}

The first thing we will discuss is whether gauge-fixing angular momentum is needed for ensuring proper commutation relations of the total canonical angular momentum operator

$$
\left[J^{i}, J^{j}\right]=\mathrm{i} \varepsilon^{i j k} J^{k} .
$$

This can be investigated with the help of (8), leading to expressions that have to be integrated by parts. They will be handled akin to the following integrals:

$$
\begin{aligned}
& \int d^{3} x d^{3} y \varepsilon^{i m n} x^{m} f(\boldsymbol{x}) g(\boldsymbol{y}) \frac{\partial}{\partial x^{n}} \delta(\boldsymbol{x}-\boldsymbol{y}) \\
& \rightarrow-\int d^{3} x \varepsilon^{i m n} x^{m}\left(\partial_{n} f\right) g
\end{aligned}
$$

and

$$
\begin{array}{r}
\int d^{3} x d^{3} y f(\boldsymbol{x}) g(\boldsymbol{y}) \frac{\partial}{\partial x^{n}} \delta(\boldsymbol{x}-\boldsymbol{y}) \\
\rightarrow \int d^{3} x\left[f \partial_{n} g-\left(\partial_{n} f\right) g\right] / 2,
\end{array}
$$

where $f$ and $g$ are some operators. It is a simple exercise to argue that no boundary terms (surface integrals) are left out in (25) and (26).

In the end, we find the following commutators [23]:

$$
\left[J_{\chi}^{i}, J_{\chi^{\prime}}^{j}\right]=\mathrm{i} \varepsilon^{i j k} J_{\chi}^{k} \delta_{\chi \chi^{\prime}} \quad \text { for } \chi, \chi^{\prime}=\text { spin, orb, } \xi,
$$

where $\delta_{\chi \chi^{\prime}}$ equals one for $\chi=\chi^{\prime}$ and zero otherwise. This shows that $\boldsymbol{J}_{\text {spin }}, \boldsymbol{J}_{\text {orb }}$, and $\boldsymbol{J}_{\boldsymbol{\xi}}$ satisfy commutation relations expected of angular momentum operators. Therefore, with or without $\boldsymbol{J}_{\xi},(24)$ is satisfied.

Finally, it is worth mentioning that (27) should not be taken for granted. This is best illustrated by the fact that Coulomb gauge versions of operators $\boldsymbol{J}_{\text {spin }}$ and $\boldsymbol{J}_{\text {orb }}$ do not satisfy such commutation relations, which came as a surprise nearly three decades ago [28]. We also mention that this well-known van Enk and Nienhuis result is discussed in review [16], where it is stated that spin and orbital canonical angular momentum operators of massless photons cannot satisfy proper angular momentum-type commutation relations. As (27) shows, such a conclusion does not apply to the covariantly quantized theory that we discuss.

\section{B. Generation of rotations}

We will take a closer look now at how various angular momentum operators contribute to generation of rotations of tensor, vector, and scalar fields relevant to our studies. So, we will investigate commutators

$$
\left[V, J_{\chi}^{i}\right]
$$

where $\chi$ is specified in (27), $V=\partial_{\mu} A_{\nu}, A_{\mu}, \partial \cdot A$, and [23] is assumed.

We start by taking $V=A_{\mu}$. On the one hand, one can show from the Lorentz-transformation properties of $A_{\mu}$ that [11]

$$
\mathrm{i}\left[A_{\mu}(z), J^{i}\right]=(z \times \nabla)^{i} A_{\mu}-\varepsilon^{i m n} \eta_{m \mu} A_{n} .
$$

On the other hand, using (8), we can decompose (29) into

$$
\begin{gathered}
\mathrm{i}\left[A_{\mu}(z), J_{\text {spin }}^{i}\right]=-\varepsilon^{i m n} \eta_{m \mu} A_{n}, \\
\mathrm{i}\left[A_{\mu}(z), J_{\text {orb }}^{i}\right]=(z \times \nabla)^{i}\left(A_{\mu}-\eta_{0 \mu} A_{0}\right), \\
\mathrm{i}\left[A_{\mu}(z), J_{\xi}^{i}\right]=\eta_{0 \mu}(z \times \nabla)^{i} A_{0} .
\end{gathered}
$$

We see from these formulas that without the gauge-fixing contribution, the total angular momentum operator does not 
generate rotations of $A_{0}$. It is so because $A_{0}$ would commute with $\boldsymbol{J}$ if $\boldsymbol{J}$ would be stripped off $\boldsymbol{J}_{\boldsymbol{\xi}}$. This observation seems to be intuitively plausible, if we take into account the fact that $A_{0}$ cannot be canonically quantized without the gaugefixing modification of the Lagrangian density.

Building on these insights, it is tempting to speculate that $\boldsymbol{J}_{\boldsymbol{\xi}}$ is only needed when rotations of fields involving $A_{0}$ are considered. Such a speculation, however, is incorrect, which we illustrate by choosing $V=\partial_{\mu} A_{\nu}$.

So, we are dealing now with

$$
\mathrm{i}\left[\partial_{\mu} A_{\nu}(z), J^{i}\right]=(z \times \boldsymbol{\nabla})^{i} \partial_{\mu} A_{\nu}+\varepsilon^{i m n}\left(\eta_{n \nu} \partial_{\mu} A_{m}-\eta_{m \mu} \partial_{n} A_{\nu}\right),
$$

which can be derived in the same way as (29). We decompose it as

$$
\begin{aligned}
\mathrm{i}\left[\partial_{\mu} A_{\nu}(z), J_{\text {spin }}^{i}\right]= & \varepsilon^{i m n} \eta_{n \nu} \partial_{\mu} A_{m}-\eta_{0 \mu} \eta_{0 \nu}(\boldsymbol{\nabla} \times \boldsymbol{A})^{i} \\
& -\varepsilon^{i m n} \eta_{0 \mu} \eta_{n \nu} \partial_{m} A_{0} \\
\mathrm{i}\left[\partial_{\mu} A_{\nu}(z), J_{\text {orb }}^{i}\right]= & (z \times \nabla)^{i}\left[\partial_{\mu} A_{\nu}+\eta_{0 \mu} \eta_{0 \nu}\left(\partial_{0} A_{0}-\operatorname{div} \boldsymbol{A}\right)\right. \\
& \left.-\eta_{0 \mu} \partial_{\nu} A_{0}-\eta_{0 \nu} \partial_{\mu} A_{0}\right] \\
& -\varepsilon^{i m n} \eta_{m \mu}\left(\partial_{n} A_{\nu}-\eta_{0 \nu} \partial_{n} A_{0}\right) \\
& +\eta_{0 \mu} \eta_{0 \nu}(\boldsymbol{\nabla} \times \boldsymbol{A})^{i} \\
\mathrm{i}\left[\partial_{\mu} A_{\nu}(z), J_{\xi}^{i}\right]= & (\boldsymbol{z} \times \boldsymbol{\nabla})^{i}\left[\eta_{0 \mu} \partial_{\nu} A_{0}+\eta_{0 \nu} \partial_{\mu} A_{0}\right. \\
& \left.+\eta_{0 \mu} \eta_{0 \nu}\left(\operatorname{div} \boldsymbol{A}-\partial_{0} A_{0}\right)\right] \\
& +\varepsilon^{i m n}\left(\eta_{0 \mu} \eta_{n \nu} \partial_{m} A_{0}-\eta_{m \mu} \eta_{0 \nu} \partial_{n} A_{0}\right)
\end{aligned}
$$

Two remarks are in order now.

First, we see from these expressions that the gauge-fixing contribution is necessary for reproducing (33). Moreover, since (36) in nonzero not only for $\nu=0$ but also for $\nu=1,2,3$, the importance of $\boldsymbol{J}_{\xi}$, in the context of rotations of field operators, cannot be restricted to expressions involving $A_{0}$ only.

Second, we easily get from (36) that

$$
\left[F_{\mu \nu}, J_{\xi}^{i}\right]=0 .
$$

This shows that manifestly gauge invariant operators, due to their sole dependence on the electromagnetic tensor $F_{\mu \nu}$, commute with $\boldsymbol{J}_{\xi}$.

Finally, regarding rotations of scalar fields, we choose $V=\partial \cdot A$ expecting on general grounds that

$$
\mathrm{i}\left[\partial \cdot A(z), J^{i}\right]=(z \times \nabla)^{i} \partial \cdot A,
$$

which we will use in Sec. III C. Straightforward calculations based on (34)-(36) lead to

$$
\begin{gathered}
{\left[\partial \cdot A, J_{\text {spin }}^{i}\right]=\left[\partial \cdot A, J_{\text {orb }}^{i}\right]=0,} \\
\mathrm{i}\left[\partial \cdot A(z), J_{\xi}^{i}\right]=(z \times \nabla)^{i} \partial \cdot A
\end{gathered}
$$

being in agreement with (38) solely thanks to $\boldsymbol{J}_{\xi}$ 's presence in $\boldsymbol{J}$. We mention in passing that vanishing of the commutator with $\boldsymbol{J}_{\text {orb }}$ should not be generalized to other scalars. For example, it follows from (31) that $\left[A_{\mu} A^{\mu}, J_{\text {orb }}^{i}\right] \neq 0$.

\section{Physical operator property}

The objective of this section is to discuss whether operators (23b)-(23d), or some of their combinations, can be classified as physical operators according to criterion (22).

The first step, however, is to make sure that the total canonical angular momentum operator satisfies such a criterion. This can be quickly verified with the help of

$$
\begin{aligned}
{\left[L_{\boldsymbol{k}}, \boldsymbol{J}\right] } & =-\mathrm{i}\left(\boldsymbol{k} \times \boldsymbol{\nabla}_{\boldsymbol{k}}\right) L_{\boldsymbol{k}}, \\
\left(\boldsymbol{\nabla}_{\boldsymbol{k}}\right)^{i} & =\partial / \partial k^{i},
\end{aligned}
$$

following from (19) and (38). Combining (18) and (41), we see that $\boldsymbol{J}$ is a physical operator. This observation agrees with common sense understanding of what the total angular momentum operator should be.

The questions now are the following. Can angular momentum operators $\boldsymbol{J}_{\text {spin }}, \boldsymbol{J}_{\text {orb }}$, and $\boldsymbol{J}_{\xi}$ also be regarded as physical operators? If not, can $\boldsymbol{J}_{\text {spin }}+\alpha \boldsymbol{J}_{\xi}$, and so also $\boldsymbol{J}_{\text {orb }}+(1-\alpha) \boldsymbol{J}_{\xi}$, be proved to be physical operators for some real $\alpha$ ? Moreover, can $\boldsymbol{J}_{\text {spin }}+\boldsymbol{J}_{\text {orb }}$, superficially representing total canonical angular momentum, be shown to be a physical operator?

To proceed, we note that by using (9) and properties of polarization vectors (12)-(16), we get

$$
\left[L_{\boldsymbol{k}}, \boldsymbol{J}_{\xi}\right]\left|\psi_{G B}\right\rangle=\frac{\mathrm{i}}{2} e^{2 \mathrm{i} \omega_{k} z^{0}}\left(\boldsymbol{k} \times \nabla_{\boldsymbol{k}}\right) L_{-\boldsymbol{k}}^{\dagger}\left|\psi_{G B}\right\rangle,
$$

$$
\begin{aligned}
& {\left[L_{\boldsymbol{k}}, \boldsymbol{J}_{\text {spin }}\right]\left|\psi_{G B}\right\rangle=} \frac{\mathrm{i}}{2 \omega_{\boldsymbol{k}}} \sum_{\sigma=1}^{2}\left[\boldsymbol{\epsilon}(\boldsymbol{k}, \sigma) \times \boldsymbol{k} c_{\boldsymbol{k} \sigma}\right. \\
&\left.+e^{2 \mathrm{i} \omega_{\boldsymbol{k}} z^{0}} \boldsymbol{k} \times \boldsymbol{\epsilon}(-\boldsymbol{k}, \sigma) c_{-\boldsymbol{k} \sigma}^{\dagger}\right]\left|\psi_{G B}\right\rangle, \\
& {\left[L_{\boldsymbol{k}}, \boldsymbol{J}_{\mathrm{orb}}\right]\left|\psi_{G B}\right\rangle=-\left[L_{\boldsymbol{k}}, \boldsymbol{J}_{\text {spin }}+\boldsymbol{J}_{\xi}\right]\left|\psi_{G B}\right\rangle, }
\end{aligned}
$$

where (44) has been obtained without using the aboveestablished fact that

$$
\left[L_{k}, J\right]\left|\psi_{G B}\right\rangle=0
$$

and $\left|\psi_{G B}\right\rangle$ satisfies (18). Several comments are in order now. 
First, $z^{0}$ dependence comes from the fact that the electromagnetic vector potential, entering definitions of angular momentum operators (23b)-(23d), depends on $z=\left(z^{0}, z\right)$.

Second, none of $\boldsymbol{J}_{\text {spin }}, \boldsymbol{J}_{\text {orb }}$, and $\boldsymbol{J}_{\boldsymbol{\xi}}$ is a physical operator

$$
\left[L_{\boldsymbol{k}}, \boldsymbol{J}_{\chi}\right]\left|\psi_{G B}\right\rangle \neq 0 \text { for } \chi=\text { spin, orb, } \boldsymbol{\xi} \text {. }
$$

Moreover, it is easy to see that no $\alpha$ leads to vanishing of $\left[L_{\boldsymbol{k}}, \boldsymbol{J}_{\text {spin }}+\alpha \boldsymbol{J}_{\xi}\right]\left|\psi_{G B}\right\rangle=-\left[L_{\boldsymbol{k}}, \boldsymbol{J}_{\text {orb }}+(1-\alpha) \boldsymbol{J}_{\xi}\right]\left|\psi_{G B}\right\rangle$.

Third, just because (42) is nonzero, the gauge-fixing contribution is necessary for making $\boldsymbol{J}$ a physical operator. This means that $\boldsymbol{J}_{\text {spin }}+\boldsymbol{J}_{\text {orb }}$ is not a physical operator in the covariantly quantized theory. All in all, answers to the above-mentioned questions are unfortunately negative.

It is perhaps worth to mention that the fact that operators $\boldsymbol{J}_{\text {spin }}, \boldsymbol{J}_{\text {orb }}$, and $\boldsymbol{J}_{\boldsymbol{\xi}}$ are gauge-variant does not imply (46). In fact, the sum of all of them, the total canonical angular momentum operator $\boldsymbol{J}$, is also gauge-variant, but it satisfies (45). We mention in passing that gauge noninvariance of $\boldsymbol{J}$ should not be surprising given the fact that we work with the gauge-fixed theory (see also [18]).

Finally, we note that

$$
\left[L_{k}, F_{\mu \nu}\right]=0 .
$$

Therefore, manifestly gauge invariant operators, in the sense mentioned below (37), can be labeled as physical.

\section{QUANTUM CONSIDERATIONS: BELINFANTE-ROSENFELD EXPRESSIONS}

We find by combining (2b), (4), (5), and (7) that the covariant-gauge quantum version of Belinfante-Rosenfeld expression (6) is

$$
\begin{gathered}
\tilde{\boldsymbol{J}}=\boldsymbol{J}_{\text {field }}+\boldsymbol{J}_{\mathrm{div}}+\boldsymbol{J}_{\xi}, \\
J_{\text {field }}^{i}=\int d^{3} z \varepsilon^{i m n} z^{m} F_{0 j} F_{j n}, \\
J_{\text {div }}^{i}=\int d^{3} z \varepsilon^{i m n} z^{m} A_{n} \partial_{j} F_{0 j} .
\end{gathered}
$$

Several remarks are in order now.

First, $\boldsymbol{J}_{\text {field }}$ is called field angular momentum to underscore its dependence on the electromagnetic field. It is given by the right-hand side of (6) stripped off the symbol cl. It is of special interest because it is gauge invariant unlike $\boldsymbol{J}_{\text {spin }}$ and $\boldsymbol{J}_{\text {orb }}$. Moreover, there is interesting classical physics associated with it (see the discussion of the Feynman's disk paradox in Secs. 17-4 and 27-6 of [29] and [30-32]).
Second, $\boldsymbol{J}_{\text {div }}$, which is absent in classical considerations (Sec. II), appears here because the covariantly quantized electric field operator satisfies the modified Gauss's law

$$
\operatorname{div} \boldsymbol{E}=-\partial_{0}(\partial \cdot A)
$$

where the right-hand side of (49) is nonzero due to (19). Nonvanishing of $\boldsymbol{J}_{\mathrm{div}}$, which is actually counterintuitive in the absence of charged particles, may be also seen as a byproduct of the fact that $\partial \cdot A=0$ cannot be imposed on the operator level. We mention in passing that the symbol $\boldsymbol{J}_{\text {div }}$ refers to the fact that there is the divergence operator in (48c).

Third, densities of the canonical and BelinfanteRosenfeld total angular momentum operators differ by the 3-divergence

$$
\boldsymbol{J}-\tilde{\boldsymbol{J}}=\int d^{3} z \partial_{j}\left[E^{j}(\boldsymbol{z} \times \boldsymbol{A})\right] .
$$

As will be seen below, the properties of $\tilde{\boldsymbol{J}}$, which we investigate, are the same as those of $\boldsymbol{J}$. Therefore, there is no need for discussion of such a boundary term in the context of our studies.

Finally, we mention that the order of operators on the right-hand sides of (48b) and (48c) does not matter, which can be verified with (9).

The following discussion will be organized similarly as in Secs. III A-III C, which will allow us to keep it concise.

\section{A. Commutation relations}

We find that commutators of angular momentum operators from (48) are given by [23]

$$
\left[J_{\text {field }}^{i}, J_{\text {field }}^{j}\right]=\mathrm{i} \varepsilon^{i j k} J_{\text {field }}^{k}+\mathrm{i} \int d^{3} z \varepsilon^{i m n} \mathcal{E}^{j k s} z^{m} z^{k} F_{s n} \operatorname{div} \boldsymbol{E},
$$

$$
\begin{gathered}
{\left[J_{\text {field }}^{i}, J_{\text {div }}^{j}\right]=-\mathrm{i} \int d^{3} z \varepsilon^{i m n} \varepsilon^{j k s} z^{m} z^{k} F_{s n} \operatorname{div} \boldsymbol{E},} \\
{\left[J_{\text {field }}^{i}, J_{\xi}^{j}\right]=0,} \\
{\left[J_{\text {div }}^{i}, J_{\text {div }}^{j}\right]=\mathrm{i} \varepsilon^{i j k} J_{\text {div }}^{k}+\mathrm{i} \int d^{3} z \varepsilon^{i m n} \varepsilon^{j k s} z^{m} z^{k} F_{s n} \operatorname{div} \boldsymbol{E},} \\
{\left[J_{\text {div }}^{i}, J_{\xi}^{j}\right]=0,}
\end{gathered}
$$

where $\left[J_{\xi}^{i}, J_{\xi}^{j}\right]$ is not listed as it is given in (27). The following observations are pertinent to these expressions.

First, there are no ordering ambiguities on the righthand sides of (51), (52), and (54), which can be shown with (9). 
Second, we see from these expressions that neither $\boldsymbol{J}_{\text {field }}$ nor $\boldsymbol{J}_{\text {div }}$ satisfies algebra expected of angular momentum operators as

$$
\left[J_{\chi}^{i}, J_{\chi^{\prime}}^{j}\right] \neq \mathrm{i} \varepsilon^{i j k} J_{\chi}^{k} \delta_{\chi \chi^{\prime}} \quad \text { for } \chi, \chi^{\prime}=\text { field, div }
$$

due to $\operatorname{div} \boldsymbol{E} \neq 0$ (49). This differs from what we have found in Sec. III A, where every component of the total angular momentum operator individually satisfied proper commutation relations.

Third, the proper commutation relations are satisfied by $\boldsymbol{J}_{\text {field }}+\boldsymbol{J}_{\text {div }}$ and $\boldsymbol{J}_{\text {field }}+\boldsymbol{J}_{\text {div }}+\boldsymbol{J}_{\xi}$. So, the total BelinfanteRosenfeld angular momentum operator does not need the $\boldsymbol{J}_{\xi}$ contribution for having proper commutation relations. It does need, however, the contribution of $\boldsymbol{J}_{\mathrm{div}}$, which is a bit surprising in the system without charged particles.

\section{B. Generation of rotations}

Proceeding similarly as in Sec. III B, we compute

$$
\begin{aligned}
\mathrm{i}\left[A_{\mu}(z), J_{\text {field }}^{i}\right]= & (z \times \nabla)^{i}\left(A_{\mu}-\eta_{0 \mu} A_{0}\right) \\
& -\varepsilon^{i m n} z^{m}\left(\partial_{\mu} A_{n}-\eta_{0 \mu} \partial_{0} A_{n}\right),
\end{aligned}
$$

$\mathrm{i}\left[A_{\mu}(z), J_{\text {div }}^{i}\right]=-\varepsilon^{i m n} \eta_{m \mu} A_{n}+\varepsilon^{i m n} z^{m}\left(\partial_{\mu} A_{n}-\eta_{0 \mu} \partial_{0} A_{n}\right)$,

and

$$
\begin{aligned}
\mathrm{i}\left[\partial_{\mu} A_{\nu}(z), J_{\text {field }}^{i}\right]= & \varepsilon^{i m n} z^{m} \partial_{\mu} F_{n \nu}-\varepsilon^{i m n} \eta_{m \mu} F_{n \nu} \\
& +\varepsilon^{i m n}\left(\eta_{0 \mu} \eta_{n \nu} F_{0 m}-\eta_{m \mu} \eta_{0 \nu} F_{0 n}\right) \\
& +\varepsilon^{i m n} z^{m}\left(\eta_{0 \mu} \partial_{\nu} F_{0 n}+\eta_{0 \nu} \partial_{\mu} F_{0 n}\right) \\
& -\varepsilon^{i m n} z^{m} \eta_{0 \mu} \eta_{0 \nu}\left(\partial_{0} F_{0 n}+\partial_{j} F_{j n}\right) \\
& +\varepsilon^{i m n} z^{m} \eta_{0 \mu} \eta_{n \nu} \partial_{j} F_{0 j}+2 \eta_{0 \mu} \eta_{0 \nu}(\nabla \times \boldsymbol{A})^{i},
\end{aligned}
$$

$$
\begin{aligned}
\mathrm{i}\left[\partial_{\mu} A_{\nu}(z), J_{\text {div }}^{i}\right]= & \varepsilon^{i m n} z^{m} \partial_{\mu} \partial_{\nu} A_{n}-\varepsilon^{i m n}\left(\eta_{m \mu} \partial_{\nu} A_{n}-\eta_{n \nu} \partial_{\mu} A_{m}\right) \\
& +\varepsilon^{i m n}\left(\eta_{m \mu} \eta_{0 \nu} \partial_{0} A_{n}-\eta_{0 \mu} \eta_{n \nu} \partial_{0} A_{m}\right) \\
& -\varepsilon^{i m n} z^{m}\left(\eta_{0 \mu} \partial_{\nu} \partial_{0} A_{n}+\eta_{0 \nu} \partial_{\mu} \partial_{0} A_{n}\right) \\
& +\varepsilon^{i m n} z^{m} \eta_{0 \mu} \eta_{0 \nu}\left(\partial_{0} \partial_{0} A_{n}+\partial_{j} \partial_{j} A_{n}\right) \\
& -\varepsilon^{i m n} z^{m} \eta_{0 \mu} \eta_{n \nu} \partial_{j} F_{0 j}-2 \eta_{0 \mu} \eta_{0 \nu}(\nabla \times \boldsymbol{A})^{i} .
\end{aligned}
$$

These two sets of results-when combined with (32) and (36) - can be used for showing that the total Belinfante-Rosenfeld angular momentum operator generates rotations of $A_{\mu}, \partial_{\mu} A_{\nu}$, and $\partial \cdot A$ (see Sec. III B for the discussion of how this can be done).

One can also show with (57)-(60) that not only $\boldsymbol{J}_{\text {div }}$ 's but also $\boldsymbol{J}_{\xi}$ 's contribution to $\tilde{\boldsymbol{J}}$ is indispensable for making it a proper generator of rotations of $A_{\mu}$ and $\partial_{\mu} A_{\nu}$. As far as rotations of $\partial \cdot A$ are concerned, we mention that $\left[\partial \cdot A, J_{\text {field }}^{i}\right]=\left[\partial \cdot A, J_{\text {div }}^{i}\right]=0$, which is very much similar to the situation encountered in Sec. III B.

All in all, it is worth stressing that $\boldsymbol{J}_{\text {field }}$ does not generate rotations of the above-discussed fields.

\section{Physical operator property}

With the help of (47), we immediately see that $\boldsymbol{J}_{\text {field }}$ is a physical operator, which agrees with common sense expectations following from its manifest gauge invariance.

The same cannot be said about $\boldsymbol{J}_{\text {div }}$, for which we get

$$
\left[L_{\boldsymbol{k}}, \boldsymbol{J}_{\mathrm{div}}\right]\left|\psi_{G B}\right\rangle=-\frac{\mathrm{i}}{2} e^{2 \mathrm{i} \omega_{k} z^{0}}\left(\boldsymbol{k} \times \boldsymbol{\nabla}_{\boldsymbol{k}}\right) L_{-\boldsymbol{k}}^{\dagger}\left|\psi_{G B}\right\rangle,
$$

where $\left|\psi_{G B}\right\rangle$ satisfies (18). Combining it with (42) and (47), we find that total Belinfante-Rosenfeld angular momentum operator (48) is a physical operator due to

$$
\left[L_{\boldsymbol{k}}, \tilde{\boldsymbol{J}}\right]\left|\psi_{G B}\right\rangle=0 .
$$

This is an expected result. What is not so obvious, however, is that it would not be the case if just one of the gaugefixing-related angular momentum operators, $\boldsymbol{J}_{\boldsymbol{\xi}}$ or $\boldsymbol{J}_{\mathrm{div}}$, would be missing in $\tilde{\boldsymbol{J}}$.

\section{PHYSICAL MATRIX ELEMENTS OF GAUGE-FIXING-RELATED OPERATORS}

Terms in above-discussed operators, which originate from the gauge-fixing modification of the Lagrangian density, have one common feature. Namely, they involve the operator $\partial \cdot A$. This is easily seen in $\boldsymbol{J}_{\xi}$ and it can be also noticed in $\boldsymbol{J}_{\text {div }}$ after employment of (49). If we now note that matrix elements of such an operator vanish in physical states of the theory (17), then one may wonder whether the very same result is obtained after replacement of $\partial \cdot A$ in (17) by a "composite" operator involving $\partial \cdot A$. If that would be the case, then physical matrix elements of either gauge-fixing-originated operators or their products with other operators could be set to zero.

The above speculation is incorrect, i.e. physical matrix elements of operators involving $\partial \cdot A$ are not necessarily zero, and we find it instructive to explain why it is so. To begin, however, we will play advocatus diaboli to get to the bottom of the issue. We consider

$$
\left\langle\psi_{G B}|O \partial \cdot A| \psi_{G B}^{\prime}\right\rangle,
$$

where $O$ is some operator, and insert between $O$ and $\partial \cdot A$ the following resolution of identity in $\mathcal{H}$ :

$$
\mathbb{1} \doteq \sum_{\left|\psi_{G B}^{\prime \prime}\right\rangle \in \mathcal{H}_{G B}}\left|\psi_{G B}^{\prime \prime}\right\rangle\left\langle\psi_{G B}^{\prime \prime}\left|+\sum_{|\phi\rangle \notin \mathcal{H}_{G B}}\right| \phi\right\rangle\langle\phi|,
$$




$$
\left\langle\psi_{G B}^{\prime \prime} \mid \phi\right\rangle \doteq 0 \quad \text { for }\left|\psi_{G B}^{\prime \prime}\right\rangle \in \mathcal{H}_{G B},|\phi\rangle \notin \mathcal{H}_{G B},
$$

where the dot over the equality symbol indicates that such an expression will be disputed below. Leaving doubts aside, we arrive at

$$
\begin{aligned}
& \left\langle\psi_{G B}|O \partial \cdot A| \psi_{G B}^{\prime}\right\rangle \\
& \quad \sum_{\left|\psi_{G B}^{\prime \prime}\right\rangle \in \mathcal{H}_{G B}}\left\langle\psi_{G B}|O| \psi_{G B}^{\prime \prime}\right\rangle\left\langle\psi_{G B}^{\prime \prime}|\partial \cdot A| \psi_{G B}^{\prime}\right\rangle \\
& \quad+\sum_{|\phi\rangle \notin \mathcal{H}_{G B}}\left\langle\psi_{G B}|O| \phi\right\rangle\left\langle\phi|\partial \cdot A| \psi_{G B}^{\prime}\right\rangle \\
& \quad \doteq 0 .
\end{aligned}
$$

The result reported in $(65 \mathrm{c})$ follows from the following observations. First, (65a) vanishes because of (17). Second, due to (19)-(21), or simply $\left[L_{k}, \partial \cdot A\right]=0$, we have $\partial \cdot A\left|\psi_{G B}^{\prime}\right\rangle \in \mathcal{H}_{G B}$, and then (65b) vanishes as a consequence of $(64 b)$.

The most elementary counterexample to the aboveobtained result is the following $[24,33]$ :

$$
\left\langle 0\left|A_{\mu}(x) \partial \cdot A(y)\right| 0\right\rangle=-\frac{\mathrm{i}}{2} \int \frac{d^{3} k}{(2 \pi)^{3}} \frac{k_{\mu}}{\omega_{k}} e^{-\mathrm{i} k \cdot(x-y)} \neq 0 .
$$

The disagreement between (65) and (66) is the result of the tacit assumption that the resolution of identity in the indefinite-metric space has the same structure as in a Hilbert space. This is actually not what is happening if one insists on the use of explicit projectors onto states from $\mathcal{H}_{G B}$, which we discuss below.

Introducing some notation, we write

$\mathbb{1}=\mathbb{1}_{0 \otimes 0}+\mathbb{1}_{1 \otimes 0}+\mathbb{1}_{0 \otimes 1}+\mathbb{1}_{2 \otimes 0}+\mathbb{1}_{1 \otimes 1}+\mathbb{1}_{0 \otimes 2}+\ldots$,

where $\mathbb{1}_{n \otimes m}$ denotes the unit operator in the subspace with the number of physical (unphysical) photons equal to $n(m)$.

In the zero-photon sector, one trivially has

$$
\mathbb{1}_{0 \otimes 0}=|0\rangle\langle 0| .
$$

In the one-photon sector, the basis states are

$$
|k \sigma\rangle=c_{\boldsymbol{k} \sigma}^{\dagger}|0\rangle,
$$

where the polarization index $\sigma=0,1,2,3$. Taking into account the fact that $\left\langle\boldsymbol{k} \sigma \mid \boldsymbol{k}^{\prime} \sigma^{\prime}\right\rangle=-\eta_{\sigma \sigma^{\prime}} \delta\left(\boldsymbol{k}-\boldsymbol{k}^{\prime}\right)$, one has

$$
\begin{gathered}
\mathbb{1}_{1 \otimes 0}=\int d^{3} k \sum_{\sigma=1}^{2}|\boldsymbol{k} \sigma\rangle\langle\boldsymbol{k} \sigma|, \\
\mathbb{1}_{0 \otimes 1}=\int d^{3} k(|\boldsymbol{k} 3\rangle\langle\boldsymbol{k} 3|-| \boldsymbol{k} 0\rangle\langle\boldsymbol{k} 0|),
\end{gathered}
$$

where the negative sign in front of the scalar-photon projector reminds us that we work in the indefinite-metric space.

To arrive at the expression that is more relevant in the context of our discussion of (64)-i.e. the one that has explicit projectors on all states from $\mathcal{H}_{G B}$-we have rewritten (71) in the following way:

$$
\begin{aligned}
\mathbb{1}_{0 \otimes 1}= & \int d^{3} k\left(a_{\alpha}\left|\Phi_{1}(\boldsymbol{k})\right\rangle\left\langle\Phi_{1}(\boldsymbol{k})\right|\right. \\
& \left.+\left|\Phi_{1}(\boldsymbol{k})\right\rangle\left\langle\mathrm{I}_{\alpha}(\boldsymbol{k})|+| \mathrm{I}_{\alpha}(\boldsymbol{k})\right\rangle\left\langle\Phi_{1}(\boldsymbol{k})\right|\right),
\end{aligned}
$$

where $\alpha \in \mathbb{C} \backslash\{-1\}$ is assumed and

$$
\begin{gathered}
\left|\Phi_{1}(\boldsymbol{k})\right\rangle=L_{\boldsymbol{k}}^{\dagger}|0\rangle=|\boldsymbol{k} 3\rangle-|\boldsymbol{k} 0\rangle, \\
\left|\mathrm{I}_{\alpha}(\boldsymbol{k})\right\rangle=\frac{|\boldsymbol{k} 3\rangle}{1+\alpha}+\frac{\alpha|\boldsymbol{k} 0\rangle}{1+\alpha}, \\
a_{\alpha}=\frac{|\alpha|^{2}-1}{|\alpha+1|^{2}} .
\end{gathered}
$$

The main difference between (71) and (72) is that the latter singles out (73), the "only" zero-norm state in the one-photon subspace of $\mathcal{H}_{G B}$ [34]. Moreover, we mention that for real $\alpha \neq-1$, the expression for $a_{\alpha}$ reduces to $(\alpha-1) /(\alpha+1)$, which is a special case of the Möbius transformation. This simple observation suggests that there might be some geometric interpretation of (72).

Next, we note that (i) the one-photon sector of $\mathcal{H}_{G B}$ is spanned by mutually orthogonal states $|\boldsymbol{k} 1\rangle,|\boldsymbol{k} 2\rangle$, and $\left|\Phi_{1}(\boldsymbol{k})\right\rangle$; (ii) $\left|\mathrm{I}_{\alpha}(\boldsymbol{k})\right\rangle \notin \mathcal{H}_{G B}$; (iii) $\left\langle\Phi_{1}(\boldsymbol{k}) \mid \mathrm{I}_{\alpha}(\boldsymbol{p})\right\rangle=$ $\delta(\boldsymbol{k}-\boldsymbol{p}) \neq 0$; (iv) states

$$
|k 1\rangle,|k 2\rangle,\left|\Phi_{1}(k)\right\rangle,\left|\mathrm{I}_{\alpha}(\boldsymbol{k})\right\rangle
$$

form a basis in the $\boldsymbol{k}$-momentum sector of the one-photon subspace of the indefinite-metric space [25].

Combining these observations, one can easily see fundamental differences between (64) and the correct resolution of identity in $\mathcal{H}$. Namely, neither (64a) matches the sum of (70) and (72) nor orthogonality condition (64b) is satisfied by all basis states (76). These unusual characteristics, absent in a Hilbert space, are not limited to the onephoton sector of the theory. We illustrate this remark in Appendix, extending the above considerations to the twophoton sector. One can also find there explanation of the steps leading to derivation of (72) and further insights into 
decomposition of unity in the indefinite-metric space of our system.

We can now apply these formulas to computation of (66). Inserting (67) between $A_{\mu}$ and $\partial \cdot A$, we instantly arrive with the help of (17) at

$$
\begin{aligned}
& \left\langle 0\left|A_{\mu}(x) \partial \cdot A(y)\right| 0\right\rangle \\
& \quad=\int d^{3} k\left\langle 0\left|A_{\mu}(x)\right| \Phi_{1}(\boldsymbol{k})\right\rangle\left\langle\mathrm{I}_{\alpha}(\boldsymbol{k})|\partial \cdot A(y)| 0\right\rangle,
\end{aligned}
$$

which reproduces (66) after straightforward algebraic manipulations.

If we now turn our attention to [18], we will find there the statement that gauge-fixing-originated contributions to operators, representing densities of canonical and Belinfante-Rosenfeld energy-momentum tensors, have vanishing physical matrix elements in covariantly quantized electrodynamics (cQED). To argue that it is so, insertion of a complete set of physical states between field operators is mentioned without an explanation of what it actually amounts to. Such a procedure of proving the above statement cannot be correct, which can be shown with the results that we have already presented in this section. We briefly comment on that below, discussing the simplest counterexample we can think of.

The free-field Feynman gauge versions of the operators discussed in [18] are [35]

$$
t_{\mathrm{can}}^{\mu \nu}(\mathrm{Gf})=-\partial \cdot A \partial^{\nu} A^{\mu}+\frac{\eta^{\mu \nu}}{2}(\partial \cdot A)^{2}
$$

and

$$
t_{\text {bel }}^{\mu \nu}(\text { Gf })=\partial^{\mu}(\partial \cdot A) A^{\nu}+\partial^{\nu}(\partial \cdot A) A^{\mu}+\frac{\eta^{\mu \nu}}{2}(\partial \cdot A)^{2} .
$$

Using (66), we find that [24]

$$
\begin{aligned}
& \left\langle 0\left|t_{\text {bel }}^{\mu \nu}(\mathrm{Gf})\right| 0\right\rangle=2\left\langle 0\left|t_{\text {can }}^{\mu \nu}(\mathrm{Gf})\right| 0\right\rangle \\
& \quad=\int \frac{d^{3} k}{(2 \pi)^{3}} \frac{k^{\mu} k^{\nu}}{\omega_{k}} \neq 0 \quad \text { for } \mu=\nu,
\end{aligned}
$$

which disagrees with (59) from [18]. Note that the fact that [18] deals with the interacting theory, cQED, does not explain this disagreement.

On the one hand, (80) illustrates the point we are trying to make that employment of the resolution of identity in the indefinite-metric space is far from trivial and it must have been misunderstood in some way in [18]. We also mention that insertion of (64a) between field operators in (78) and (79) sets (80) to zero, reproducing the result reported in [18]. The same wrong null result is obtained if one inserts, during computation of (80), only the first term of (64a) between the field operators.
On the other hand, (80) suggests reexamination of conclusions first discussed by the end of Sec. IV of [18] and then reiterated in Sec. 3.1.1 of [15].

If we now turn our attention to angular momentum operators involving $\partial \cdot A$, we will find that

$$
\left\langle\psi_{G B}\left|\boldsymbol{J}_{\xi}\right| \psi_{G B}^{\prime}\right\rangle=\left\langle\psi_{G B}\left|\boldsymbol{J}_{\mathrm{div}}\right| \psi_{G B}^{\prime}\right\rangle=0 .
$$

This is seen by using (9)-(21) to show that

$$
\begin{aligned}
\left\langle\psi_{G B}\left|\partial \cdot A \partial_{n} A_{0}\right| \psi_{G B}^{\prime}\right\rangle & =-\left\langle\psi_{G B}\left|A_{n} \partial_{0}(\partial \cdot A)\right| \psi_{G B}^{\prime}\right\rangle \\
& =\left\langle\psi_{T} \mid \psi_{T}^{\prime}\right\rangle \int \frac{d^{3} k}{(2 \pi)^{3}} \frac{k^{n}}{2}=0,
\end{aligned}
$$

where $\left|\psi_{G B}^{\prime}\right\rangle$ and $\left|\psi_{T}^{\prime}\right\rangle$ are related to each other via the "primed" version of (20).

It should be now understood that (81) is obtained under the assumption that the free electromagnetic field is considered. In the interacting theory, $\mathrm{CQED}$, results such as (81) give tree-level (zeroth-order) expressions and perturbative (loop) calculations are needed for checking whether there are radiative corrections to them. For example, a one-loop correction to gauge-fixing angular momentum, in the cQED ground state describing the electron at rest, depends on [36]

$$
\begin{aligned}
\langle 0| \mathbb{T} & :\left(\boldsymbol{J}_{\xi}^{I}\right)^{i}: A_{\mu}^{I}(x) A_{\nu}^{I}(y)|0\rangle \\
= & \int d^{3} z \varepsilon^{i m n} z^{m}\left\langle 0\left|\mathbb{T}: \partial \cdot A_{I}(z) \partial_{n} A_{0}^{I}(z): A_{\mu}^{I}(x) A_{\nu}^{I}(y)\right| 0\right\rangle \\
= & \int \frac{d^{3} z d^{4} p d^{4} q}{(2 \pi)^{8}} \frac{\varepsilon^{i m n} z^{m} p_{\mu} q_{n} \eta_{0 \nu}}{\left(p^{2}+\mathrm{i} 0\right)\left(q^{2}+\mathrm{i} 0\right)} e^{\mathrm{i} p \cdot(z-x)+\mathrm{i} q \cdot(z-y)} \\
& +(x, \mu \leftrightarrow y, \nu),
\end{aligned}
$$

where $\mathbb{T}$ is the time-ordering operator, : : denotes normal ordering, and $A_{I}^{\mu}$ is the interaction-picture vector potential, which can be written just as (9). The answer to the question, whether one-loop radiative corrections make the expectation value of $\boldsymbol{J}_{\boldsymbol{\xi}}$ nonzero, relies then on fermionic contributions, which are contracted with (83). It is shown in [36] that $\left\langle\boldsymbol{J}_{\xi}\right\rangle$ is nonzero in the cQED ground state describing the electron at rest. It is also shown there that such a gauge-fixing contribution is needed in order to obtain the result $\left\langle\boldsymbol{J}_{\text {total }}\right\rangle=\hat{\boldsymbol{s}} / 2+O\left(\alpha^{2}\right)$ for the electron's total angular momentum in such a state, where $\boldsymbol{J}_{\text {total }}$ stands for the total canonical angular momentum operator of cQED, the unit 3-vector $\hat{\boldsymbol{s}}$ denotes polarization of the electron's spin, $\alpha$ is the fine-structure constant, and $O\left(\alpha^{2}\right)$ reflects the fact that two- and higher-loop radiative corrections were not studied in [36]. 


\section{SUMMARY}

If one wants to study the total angular momentum operator of the free electromagnetic field, then the traditional discussion of angular momentum decompositions makes the impression that one has to choose between the canonical

$$
\boldsymbol{J}_{\text {spin }}+\boldsymbol{J}_{\text {orb }},
$$

the Belinfante-Rosenfeld

$$
\boldsymbol{J}_{\text {field }} \text {, }
$$

or some other expressions free from explicit gauge-fixingoriginated contributions (see e.g. review [16]). The reality, however, is that in the covariantly quantized theory neither (84) nor (85) can be considered as a total angular momentum operator.

This has been discussed in Secs. III and IV, where it has been shown that neither (84) nor (85) generates rotations of quantum fields. It can be also found there that these expressions have some complementary deficiencies. For example, (84) has an unwelcome feature that it takes physical states of the theory outside of the physical sector of the state space, whereas (85) does not lead to such complications. At the same time, (85) does not satisfy commutation relations expected of a total angular momentum operator while (84) does. These are basic reasons why a genuine total angular momentum operator of the covariantly quantized electromagnetic field, such as (23) or (48), needs the explicit gauge-fixing contribution(s).

It should be also recognized that gauge-fixing is implicitly encoded in (84) and (85) once these operators are defined via the vector potential, which satisfies commutation relations (8) or has Fourier expansion (9). It is so because both (8) and (9) owe their elegant covariant form to the gauge-fixing modification of the Lagrangian density. Looking from this perspective, the need for inclusion of gauge-fixing corrections to (84) and (85) can be seen as a consistency requirement. Namely, one should not benefit from gauge-fixing when it comes to field operators and pretend that it is nonexistent while investigating total angular momentum operators.

The above discussion can be easily extended to quantum electrodynamics, where physical insights are typically gained via perturbative expansions as it is an interacting theory. Those are oftentimes carried out with the following interaction-picture photon propagator:

$$
\left\langle 0\left|\mathbb{T} A_{\mu}^{I}(x) A_{\nu}^{I}(y)\right| 0\right\rangle=\int \frac{d^{4} k}{(2 \pi)^{4}} \frac{-\mathrm{i} \eta_{\mu \nu}}{k^{2}+\mathrm{i} 0} e^{-\mathrm{i} k \cdot(x-y)},
$$

having such an elegant covariant form due to the gaugefixing modification of the Lagrangian density. Thus, employment of (86) in actual calculations automatically implies that the Lagrangian is gauge fixed and that some observables derived from it acquire gauge-fixing-originated contributions absent in the classical theory.

Our studies should not suggest that only those angular momentum operators that satisfy all criteria investigated in Secs. III and IV are physically interesting. In fact, there are different forms of angular momentum. The operators representing them cannot possibly have the same properties as a total angular momentum operator, if they are just a part of it. Still, they can be in principle measurable and carry out useful information about the studied system (see e.g. $[16,28]$ for a similar viewpoint). For this to happen, they do not need to have angular momentum-like commutation relations and do not have to properly generate rotations. We assume, however, that they should satisfy physical observable criterion. As far as this work is concerned, (85) is an example of such an operator, which is additionally also gauge invariant (manifest gauge invariance of $\boldsymbol{J}_{\text {field }}$ actually implies its physical operator property). We mention in passing that its studies in CQED can be found in [37], see also [38].

Having mentioned gauge invariance, it should be also said that total angular momentum operators, (23) and (48), are gauge variant. This is not a controversial feature because gauge-fixing explicitly breaks invariance of the theory with respect to arbitrary gauge transformations (see also discussion in [18]). Note that this does not mean that matrix elements of such operators, in physical states of the theory, are gauge variant. For example, it was explicitly shown in [36] that the one-loop expectation value of the total canonical angular momentum operator, in the cQED state describing the electron at rest, equals $1 / 2$ in all covariant gauges.

These remarks bring us to the discussion of physical matrix elements of the so-called gauge-fixing-related operators, i.e., the ones involving $\partial \cdot A$ in the context of our studies. We have shown how one can reach incorrect results for such matrix elements, if one does not take into account subtleties of the resolution of identity in the indefinitemetric space of the covariantly quantized electromagnetic field. Namely, one has to be careful when one wants to use explicit projectors onto the physical sector of such a space. If this is the case, then not-so-intuitive expressions for the unit operator, in the one- and two-photon subspaces, take the form discussed in Sec. V and Appendix. Apart from technical insights, these considerations convey the message that it should not be a priori assumed that physical matrix elements of operators, having gauge-fixing-related components, vanish. It would be interesting to investigate similar issues in quantum chromodynamics.

\section{ACKNOWLEDGMENTS}

This work has been supported by the Polish National Science Centre (NCN) Grant No. 2019/35/B/ST2/00034. 


\section{APPENDIX: TWO-PHOTON SECTOR OF $\mathbb{1}$}

We discuss here the resolution of identity in the twophoton subspace of the indefinite-metric space $\mathcal{H}$. On the one hand, this allows for explicit illustration of the fact that the abnormal form of the unit operator, which we have encountered in Sec. V, is not specific to the one-photon sector of $\mathcal{H}$. On the other hand, the following studies clearly illustrate the logic behind derivation of (72), which has not been explained in Sec. V. Some general remarks about our studies of the resolution of identity are provided by the end of this appendix.

The two-physical-photon projector is given by

$$
\begin{aligned}
\mathbb{1}_{2 \otimes 0} & =\int d^{3} k d^{3} k^{\prime} \sum_{\sigma, \sigma^{\prime}=1}^{2}\left|\boldsymbol{k} \sigma \boldsymbol{k}^{\prime} \sigma^{\prime}\right\rangle\left\langle\boldsymbol{k} \sigma \boldsymbol{k}^{\prime} \sigma^{\prime}\right|, \\
\left|\boldsymbol{k} \sigma \boldsymbol{k}^{\prime} \sigma^{\prime}\right\rangle & =\frac{1}{\sqrt{2}} c_{\boldsymbol{k} \sigma}^{\dagger} c_{\boldsymbol{k}^{\prime} \sigma^{\prime}}^{\dagger}|0\rangle .
\end{aligned}
$$

The mixed physical-unphysical-photon projector reads

$$
\mathbb{1}_{1 \otimes 1}=\int d^{3} k^{\prime} \sum_{\sigma^{\prime}=1}^{2} c_{\boldsymbol{k}^{\prime} \sigma^{\prime}}^{\dagger} \mathbb{1}_{0 \otimes 1} c_{\boldsymbol{k}^{\prime} \sigma^{\prime}} .
$$

A more interesting situation is encountered in the twounphysical-photon sector, where

$$
\begin{aligned}
\mathbb{1}_{0 \otimes 2}= & \int d^{3} k d^{3} k^{\prime}\left(\left|\boldsymbol{k} 3 \boldsymbol{k}^{\prime} 3\right\rangle\left\langle\boldsymbol{k} 3 \boldsymbol{k}^{\prime} 3|+| \boldsymbol{k} 0 \boldsymbol{k}^{\prime} 0\right\rangle\left\langle\boldsymbol{k} 0 \boldsymbol{k}^{\prime} 0\right|\right. \\
& \left.-\left|\boldsymbol{k} 0 \boldsymbol{k}^{\prime} 3\right\rangle\left\langle\boldsymbol{k} 0 \boldsymbol{k}^{\prime} 3|-| \boldsymbol{k} 3 \boldsymbol{k}^{\prime} 0\right\rangle\left\langle\boldsymbol{k} 3 \boldsymbol{k}^{\prime} 0\right|\right) .
\end{aligned}
$$

In this sector of the indefinite-metric space, there is one state from $\mathcal{H}_{G B}$, the zero-norm state (A4). Our goal now is to rewrite (A3) such that it contains explicit projection onto it. This will complete our efforts towards decomposition of the unit operator (in the two-photon sector) into explicit projectors onto all states from the physical subspace of the theory.

So, we start calculations with

$$
\begin{aligned}
\left|\Phi_{2}\left(\boldsymbol{k}, \boldsymbol{k}^{\prime}\right)\right\rangle & =\frac{\beta}{2} L_{\boldsymbol{k}}^{\dagger} L_{\boldsymbol{k}^{\prime}}^{\dagger}|0\rangle \\
& =\frac{\beta}{\sqrt{2}}\left(\left|\boldsymbol{k} 3 \boldsymbol{k}^{\prime} 3\right\rangle+\left|\boldsymbol{k} 0 \boldsymbol{k}^{\prime} 0\right\rangle-\left|\boldsymbol{k} 3 \boldsymbol{k}^{\prime} 0\right\rangle-\left|\boldsymbol{k} 0 \boldsymbol{k}^{\prime} 3\right\rangle\right),
\end{aligned}
$$

where the normalizing prefactor $\beta$ will be determined later on [39]. Without loss of generality, real $\beta \neq 0$ will be considered below.

Then, we find two states with two unphysical photons having $\boldsymbol{k}$ and $\boldsymbol{k}^{\prime}$ momenta, which are not only mutually orthogonal but also orthogonal to (A4) and linearly independent from it

$$
\begin{aligned}
& \left|\mathrm{II}^{\prime}\left(\boldsymbol{k}, \boldsymbol{k}^{\prime}\right)\right\rangle=\frac{1}{\sqrt{2}}\left(\left|\boldsymbol{k} 3 \boldsymbol{k}^{\prime} 3\right\rangle-\left|\boldsymbol{k} 0 \boldsymbol{k}^{\prime} 0\right\rangle\right), \\
& \left|\mathrm{II}^{\prime \prime}\left(\boldsymbol{k}, \boldsymbol{k}^{\prime}\right)\right\rangle=\frac{1}{\sqrt{2}}\left(\left|\boldsymbol{k} 3 \boldsymbol{k}^{\prime} 0\right\rangle-\left|\boldsymbol{k} 0 \boldsymbol{k}^{\prime} 3\right\rangle\right) .
\end{aligned}
$$

Their normalization is chosen such that the projectors

$$
\begin{aligned}
P^{\prime} & =\int d^{3} k d^{3} k^{\prime}\left|\mathrm{II}^{\prime}\left(\boldsymbol{k}, \boldsymbol{k}^{\prime}\right)\right\rangle\left\langle\mathrm{II}^{\prime}\left(\boldsymbol{k}, \boldsymbol{k}^{\prime}\right)\right|, \\
P^{\prime \prime} & =\int d^{3} k d^{3} k^{\prime}\left|\mathrm{II}^{\prime \prime}\left(\boldsymbol{k}, \boldsymbol{k}^{\prime}\right)\right\rangle\left\langle\mathrm{II}^{\prime \prime}\left(\boldsymbol{k}, \boldsymbol{k}^{\prime}\right)\right|
\end{aligned}
$$

satisfy $\quad P^{\prime}\left|\mathrm{II}^{\prime}\left(\boldsymbol{p}, \boldsymbol{p}^{\prime}\right)\right\rangle=\left|\mathrm{II}^{\prime}\left(\boldsymbol{p}, \boldsymbol{p}^{\prime}\right)\right\rangle \quad$ and $\quad P^{\prime \prime}\left|\mathrm{II}^{\prime \prime}\left(\boldsymbol{p}, \boldsymbol{p}^{\prime}\right)\right\rangle=$ $-\left|\mathrm{II}^{\prime \prime}\left(\boldsymbol{p}, \boldsymbol{p}^{\prime}\right)\right\rangle$. These two states do not belong to $\mathcal{H}_{G B}$.

Next, we find a family of states labeled by the parameter $\alpha \in \mathbb{C} \backslash\{-1\}$, which are distinct (linearly independent) from $\left|\Phi_{2}\left(\boldsymbol{k}, \boldsymbol{k}^{\prime}\right)\right\rangle$ and orthogonal to both $\left|\mathrm{II}^{\prime}\left(\boldsymbol{k}, \boldsymbol{k}^{\prime}\right)\right\rangle$ and $\left|\mathrm{II}^{\prime \prime}\left(\boldsymbol{k}, \boldsymbol{k}^{\prime}\right)\right\rangle$ :

$$
\left|\mathrm{II}_{\alpha}\left(\boldsymbol{k}, \boldsymbol{k}^{\prime}\right)\right\rangle=\gamma\left(\left|\boldsymbol{k} 3 \boldsymbol{k}^{\prime} 3\right\rangle+\left|\boldsymbol{k} 0 \boldsymbol{k}^{\prime} 0\right\rangle+\alpha\left|\boldsymbol{k} 3 \boldsymbol{k}^{\prime} 0\right\rangle+\alpha\left|\boldsymbol{k} 0 \boldsymbol{k}^{\prime} 3\right\rangle\right),
$$

where the normalizing factor $\gamma \in \mathbb{C} \backslash\{0\}$ will be fixed in (A11). Such states also do not belong to $\mathcal{H}_{G B}$.

We use them to build the projector onto $\left|\Phi_{2}\left(\boldsymbol{k}, \boldsymbol{k}^{\prime}\right)\right\rangle$, which cannot be written as $\int d^{3} k d^{3} k^{\prime}\left|\Phi_{2}\left(\boldsymbol{k}, \boldsymbol{k}^{\prime}\right)\right\rangle\left\langle\Phi_{2}\left(\boldsymbol{k}, \boldsymbol{k}^{\prime}\right)\right|$ due to the zero norm of such a state. Namely, we introduce

$$
\begin{aligned}
P_{\alpha}= & \int d^{3} k d^{3} k^{\prime}\left(\left|\Phi_{2}\left(\boldsymbol{k}, \boldsymbol{k}^{\prime}\right)\right\rangle\left\langle\mathrm{II}_{\alpha}\left(\boldsymbol{k}, \boldsymbol{k}^{\prime}\right)\right|\right. \\
& \left.+\left|\mathrm{II}_{\alpha}\left(\boldsymbol{k}, \boldsymbol{k}^{\prime}\right)\right\rangle\left\langle\Phi_{2}\left(\boldsymbol{k}, \boldsymbol{k}^{\prime}\right)\right|\right)
\end{aligned}
$$

and normalize (A9) by choosing

$$
\gamma=\frac{1}{\sqrt{2} \beta(1+\alpha)}
$$

so that $P_{\alpha}\left|\Phi_{2}\left(\boldsymbol{p}, \boldsymbol{p}^{\prime}\right)\right\rangle=\left|\Phi_{2}\left(\boldsymbol{p}, \boldsymbol{p}^{\prime}\right)\right\rangle$. For this to happen, one actually does not need the second term on the right-hand side of (A10), which is added to ensure Hermiticity of such a projector.

By comparing (A3) and $P_{\alpha}+P^{\prime}-P^{\prime \prime}$, we find that the difference between the two is given by $\int d^{3} k d^{3} k^{\prime} a_{\alpha} \beta^{-2}\left|\Phi_{2}\left(\boldsymbol{k}, \boldsymbol{k}^{\prime}\right)\right\rangle\left\langle\Phi_{2}\left(\boldsymbol{k}, \boldsymbol{k}^{\prime}\right)\right|$, where $a_{\alpha}$ has been already introduced in (75).

In the end, making use of the freedom to choose $\beta$, we set

$$
\beta=1
$$

getting 


$$
\begin{aligned}
\mathbb{1}_{0 \otimes 2}= & \int d^{3} k d^{3} k^{\prime}\left(a_{\alpha}\left|\Phi_{2}\left(\boldsymbol{k}, \boldsymbol{k}^{\prime}\right)\right\rangle\left\langle\Phi_{2}\left(\boldsymbol{k}, \boldsymbol{k}^{\prime}\right)\right|\right. \\
& +\left|\Phi_{2}\left(\boldsymbol{k}, \boldsymbol{k}^{\prime}\right)\right\rangle\left\langle\mathrm{II}_{\alpha}\left(\boldsymbol{k}, \boldsymbol{k}^{\prime}\right)|+| \mathrm{II}_{\alpha}\left(\boldsymbol{k}, \boldsymbol{k}^{\prime}\right)\right\rangle\left\langle\Phi_{2}\left(\boldsymbol{k}, \boldsymbol{k}^{\prime}\right)\right| \\
& \left.+\left|\mathrm{II}^{\prime}\left(\boldsymbol{k}, \boldsymbol{k}^{\prime}\right)\right\rangle\left\langle\mathrm{II}^{\prime}\left(\boldsymbol{k}, \boldsymbol{k}^{\prime}\right)|-| \mathrm{II}^{\prime \prime}\left(\boldsymbol{k}, \boldsymbol{k}^{\prime}\right)\right\rangle\left\langle\mathrm{II}^{\prime \prime}\left(\boldsymbol{k}, \boldsymbol{k}^{\prime}\right)\right|\right) .
\end{aligned}
$$

Two remarks are in order now.

First, thanks to (A12), the coefficient in front of $\left|\Phi_{2}\right\rangle\left\langle\Phi_{2}\right|$ in (A13) is the same as the one in front of $\left|\Phi_{1}\right\rangle\left\langle\Phi_{1}\right|$ in (72). Such a choice facilitates comparison between the two expressions.

Second, the fact that (A13) is built of four linearly independent vectors- $\left|\Phi_{2}\right\rangle,\left|\mathrm{II}_{\alpha}\right\rangle,\left|\mathrm{II}^{\prime}\right\rangle$, and $\left|\mathrm{II}^{\prime \prime}\right\rangle$-reflects the number of ways one can distribute two unphysical photons into two momentum modes.

Looking now back at the discussion from Sec. V, one can easily check that the same scheme has been utilized there for decomposition of unity in the one-photon sector. Expressions from Sec. V, however, are a bit simpler because there are no single-unphysical-photon equivalents of $\left|\mathrm{II}^{\prime}\right\rangle$ and $\left|\mathrm{II}^{\prime \prime}\right\rangle$ that are orthogonal to $\left|\Phi_{1}\right\rangle$.

We expect that the above-explained strategy for construction of (A1), (A2), and (A13) can be generalized so as to yield the unit operator in higher photon-number sectors with explicit projectors onto all states belonging to $\mathcal{H}_{G B}$. Such studies, however, are beyond the scope of this work.

To put our results in a broader perspective, we have the following remarks. Our decomposition of the unit operator is built of eigenstates of the normal-ordered
Hamiltonian describing the covariantly quantized free electromagnetic field [11]

$$
H=\int d^{3} k \omega_{k}\left(c_{k 1}^{\dagger} c_{k 1}+c_{k 2}^{\dagger} c_{k 2}+c_{k 3}^{\dagger} c_{k 3}-c_{k 0}^{\dagger} c_{k 0}\right) .
$$

While working in the one- and two-photon sectors, we make use of its degenerate eigenstates. Namely, $|\boldsymbol{k} \sigma=1,2\rangle$, $\left|\Phi_{1}(\boldsymbol{k})\right\rangle,\left|\mathrm{I}_{\alpha}(\boldsymbol{k})\right\rangle$ in the one-photon sector and $\mid \boldsymbol{k} \sigma=1$, $\left.2 \boldsymbol{k}^{\prime} \sigma^{\prime}=1,2\right\rangle,\left|\Phi_{2}\left(\boldsymbol{k}, \boldsymbol{k}^{\prime}\right)\right\rangle,\left|\mathrm{II}_{\alpha}\left(\boldsymbol{k}, \boldsymbol{k}^{\prime}\right)\right\rangle$, etc. in the twophoton sector. Degeneracy of these eigenstates follows from

$$
\begin{gathered}
H|\boldsymbol{k} \sigma\rangle=\omega_{\boldsymbol{k}}|\boldsymbol{k} \sigma\rangle, \\
H\left|\boldsymbol{k} \sigma \boldsymbol{k}^{\prime} \sigma^{\prime}\right\rangle=\left(\omega_{\boldsymbol{k}}+\omega_{\boldsymbol{k}^{\prime}}\right)\left|\boldsymbol{k} \sigma \boldsymbol{k}^{\prime} \sigma^{\prime}\right\rangle,
\end{gathered}
$$

which are valid for all $\sigma, \sigma^{\prime}=0,1,2,3$.

The question how to construct the unit operator, out of eigenstates of a Hermitian operator in the indefinite-metric space, is in general quite nontrivial [4,5]. An algorithm for achieving this goal in finite-dimensional systems, from eigenstates of operators having nondegenerate spectrum, is discussed in Sec. 1.2 of [4]. It is similar to our approach in the sense that it also involves projectors onto zero-norm states. The details, however, are quite different presumably due to the fact that we work with degenerate eigenstates. Taking also into account that our space is actually infinite dimensional, we see our results as complementary to those reported in [4].
[1] T. Kugo and I. Ojima, Prog. Theor. Phys. Suppl. 66, 1 (1979).

[2] J. Schwinger, Phys. Rev. 115, 721 (1959).

[3] F. Strocchi, Phys. Rev. D 2, 2334 (1970).

[4] K. L. Nagy, State Vector Spaces with Indefinite Metric in Quantum Field Theory (Akadémiai Kiadó, Budapest, 1966).

[5] N. Nakanishi, Prog. Theor. Phys. Suppl. 51, 1 (1972).

[6] For any vector $|\psi\rangle$, we define the norm to be given by the inner product $\langle\psi \mid \psi\rangle$.

[7] S. N. Gupta, Proc. Phys. Soc. London Sect. A 63, 681 (1950).

[8] K. Bleuler, Helv. Phys. Acta 23, 567 (1950).

[9] S. N. Gupta, Quantum Electrodynamics (Gordon and Breach Science Publishers, New York, 1977).

[10] B. Lautrup, Mat. Fys. Medd. K. Dan. Vidensk. Selsk 35, No. 11 (1967).

[11] R. Greiner and J. Reinhardt, Field Quantization (SpringerVerlag, Berlin, 1996).

[12] D. N. Blaschke and F. Gieres, Nucl. Phys. B965, 115366 (2021).
[13] S. Weinberg, The Quantum Theory of Fields, Modern Applications Vol. II (Cambridge University Press, Cambridge, England, 1996).

[14] F. Strocchi, Phys. Rev. 162, 1429 (1967).

[15] E. Leader and C. Lorcé, Phys. Rep. 541, 163 (2014); 802, 23(E) (2019).

[16] M. Wakamatsu, Int. J. Mod. Phys. A 29, 1430012 (2014).

[17] The term gauge-fixing appears in Ref. [16] in a different context. It refers there to "a process of eliminating unphysical gauge degrees of freedom, thereby selecting out physical degrees of freedom of the gauge field." In our paper, gauge-fixing terminology refers to the $-(\partial \cdot A)^{2} / 2$ modification of the Lagrangian density and its impact on angular momentum of the electromagnetic field.

[18] E. Leader, Phys. Rev. D 83, 096012 (2011).

[19] G. S. Adkins, Phys. Rev. D 27, 1814 (1983).

[20] F. J. Belinfante, Physica (Amsterdam) 6, 887 (1939).

[21] L. Rosenfeld, in Selected Papers of Léon Rosenfeld, edited by R. S. Cohen and J. J. Stachel (D. Reidel, Dordrecht, 1979), p. 711-735. 
[22] F. J. Belinfante, Physica (Amsterdam) 7, 449 (1940).

[23] All commutators in this paper, which involve two field operators, two angular momentum operators, or a field and an angular momentum operator, are computed under the equal-time assumption.

[24] All integrals of the form $\int d^{3} k f(k)$, where $f$ is an arbitrary function, are written under the assumption that $k^{0}=\omega_{k}$.

[25] All expressions where some 3-momentum is singled out, just as $\boldsymbol{k}$ in Ref. (18), are supposed to hold for all such 3-momenta.

[26] R. Haag, Local Quantum Physics, 2nd ed. (Springer-Verlag, Berlin, 1996).

[27] F. Strocchi and A. S. Wightman, J. Math. Phys. (N.Y.) 15, 2198 (1974).

[28] S. J. van Enk and G. Nienhuis, Europhys. Lett. 25, 497 (1994); J. Mod. Opt. 41, 963 (1994).

[29] R. P. Feynman, R. B. Leighton, and M. Sands, The Feynman Lectures on Physics, Mainly Electromagnetism and Matter Vol. II (Addison-Wesley, Reading, MA, 1964).

[30] G. G. Lombardi, Am. J. Phys. 51, 213 (1983).

[31] T. Bahder and J. Sak, Am. J. Phys. 53, 495 (1985).

[32] T. E. Ma, Am. J. Phys. 54, 949 (1986).

[33] The same expectation value was used in Ref. [3] for illustration of the inconsistency of the so-called Fermi condition, incorrectly identifying physical states of the covariantly quantized electromagnetic field.

[34] The quotation marks refer to the facts that (i) (73) actually represents a whole family of zero-norm states labeled by the momentum $\boldsymbol{k}$, and (ii) there is no unique normalization of zero-norm states.

[35] To compare our (78) and (79) to (55) and (58) from Ref. [18], one has to use field equations, which in the Feynman gauge version relevant to our studies, identify $B$, the gauge-fixing field from Ref. [18], with $-\partial \cdot A$.

[36] B. Damski, Nucl. Phys. B955, 115042 (2020).

[37] B. Damski, Nucl. Phys. B949, 114828 (2019).

[38] Regularization ambiguities discussed in Ref. [37] are resolved by the systematic approach developed in [36], which consistently imposes Pauli-Villars regularization across all calculations. It shows that ad hoc Pauli-Villars regularization attempts explored in Ref. [37] lead to the correct one-loop result for field angular momentum of the electron.

[39] Strictly speaking, one cannot normalize a zero-norm vector such as $\left|\Phi_{2}\right\rangle$. One can, however, fix the overall prefactor of such a state, which is what we do. 